\title{
Study of Virtual Remote Diagnosis System of Printing Equipment
}

\author{
Xiong Wei ${ }^{1}$, Chikun Gong ${ }^{1}$, Baojiang $\mathrm{Du}^{1}$, Jingmin Guo ${ }^{1}$ \& Changqin $\mathrm{Ji}^{1}$ \\ ${ }^{1}$ College of Mechanical Engineering, University of Shanghai for Science and Technology, Shanghai, China \\ Correspondence: Xiong Wei, College of Mechanical Engineering, University of Shanghai for Science and \\ Technology, 516 Jun Gong Road, Shanghai 200093, China. Tel: 86-137-8898-4142. E-mail: \\ weixiong1234@sina.com
}

$\begin{array}{lc}\text { Received: April 18, } 2012 & \text { Accepted: May 3, } 2012 \quad \text { Online Published: May 25, } 2012 \\ \text { doi:10.5539/mer.v2n1p76 } & \text { URL: http://dx.doi.org/10.5539/mer.v2n1p76 }\end{array}$

The project of science and technology commission of Shanghai municipality: Study of virtual manufacturing technology and service model for equipments (11DZ1121300)

\begin{abstract}
In this paper, the structure and relative function of virtual remote diagnosis system of printing equipment was presented. Its network structure is based on 3G, which is advanced wireless network technology at present. Visiting internal data of Programmable Logic Controller (PLC) which controls the printing equipment is the way to get parameters of equipment. Driving virtual prototype is in order to simulate work in real time. Analyzing these parameters via experience database which was designed reasonably and logically is in order to maintain the equipmentand detect themal function timely by the virtual remote diagnosis system.
\end{abstract}

Keywords: virtual prototype, experience database, virtual remote diagnosis

\section{Introduction}

With the development of science and technology and modern industry, the printing equipment is larger, more automatic and more complicated. It demands to maintain the equipment strictly. Improving the response time and the efficiency of maintenance, the efficiency and quality of sales service will bein favor of reliable operation of protective equipment and personal safety. Virtual remote diagnosis and maintenance system into the after-sales service and technical support links for manufacturers with a very significance.

On actual remote malfunction diagnosis, device information returned by the remote is large amounts of data. Technicians need tounderstand theabstract operation status to analyze the reasons of malfunction of equipment. It greatly increases the mental workload of technicians, impact efficiency to solve malfunction problems, reduces the after sale service response speed and service quality. In addition to delaying the enterprise information management upgrade, it does nothelp to improve the market competitiveness of enterprises.

The virtual remote diagnosis system can effectively solve theproblem. It obtains the status of the device to analyze the cause of the malfunction before technician will be dispatch. According to the specific issues, it develops solutions to improve the response speed ofthe service. It can effectively reduce energy consumption and cost of servicesby reducing the dispatch of blindness. Especially in the application system sub-Ministry of the vast territory, it can reduce service costs, and system integrators in the market competition advantage.

\section{Virtual Remote Diagnosis System}

\subsection{Analysis of Virtual Remote Diagnostic System}

Virtual remote malfunction diagnosis System uses PLC of the printing equipment as a mechanical malfunction of data acquisition and processing tools. Virtual manufacturing related technologies and other related technologies based on 3G wireless data communication technology, database technology, virtual reality technology to solve for the PLC control of the core machinery and equipment of remote malfunction diagnosis and maintenance issues. The failure of the remote handling equipment, the technology in real-time operational status of the analog field devices, in order to diagnose correctly, intuitive grasp of the operation of equipment, a comprehensive analysis quickly make the correct malfunction diagnosis decision-making.

Virtual remote malfunction diagnosis and maintenance solutions, when the acquisition of PLC data remotely via 
a wireless communication network is divided into two categories, one category is machinery and equipment operating data, including the speed of the motor current and voltage of components, equipment and work efficiency fordrive a virtual devices running in real time; other mechanical equipment failure data for the detection database for suspicious point of failure. The diagnosis to malfunction diagnosis, the more suspicious point of failure with the virtual device operating conditions, the relationship of the adjoining parts of the suspicious point of failure location, sports relations, control information such as results of a comprehensive analysis of equipment failure, arrive at a diagnosis results and the corresponding failure to maintain.

\subsection{Virtual Remote Function of Each}

There are four aspects of the system functional modules in this system, such as the remote data communications, real-time virtual device driver, database management, virtual malfunction diagnosis and maintenance. The Figure 1 shows four aspects.

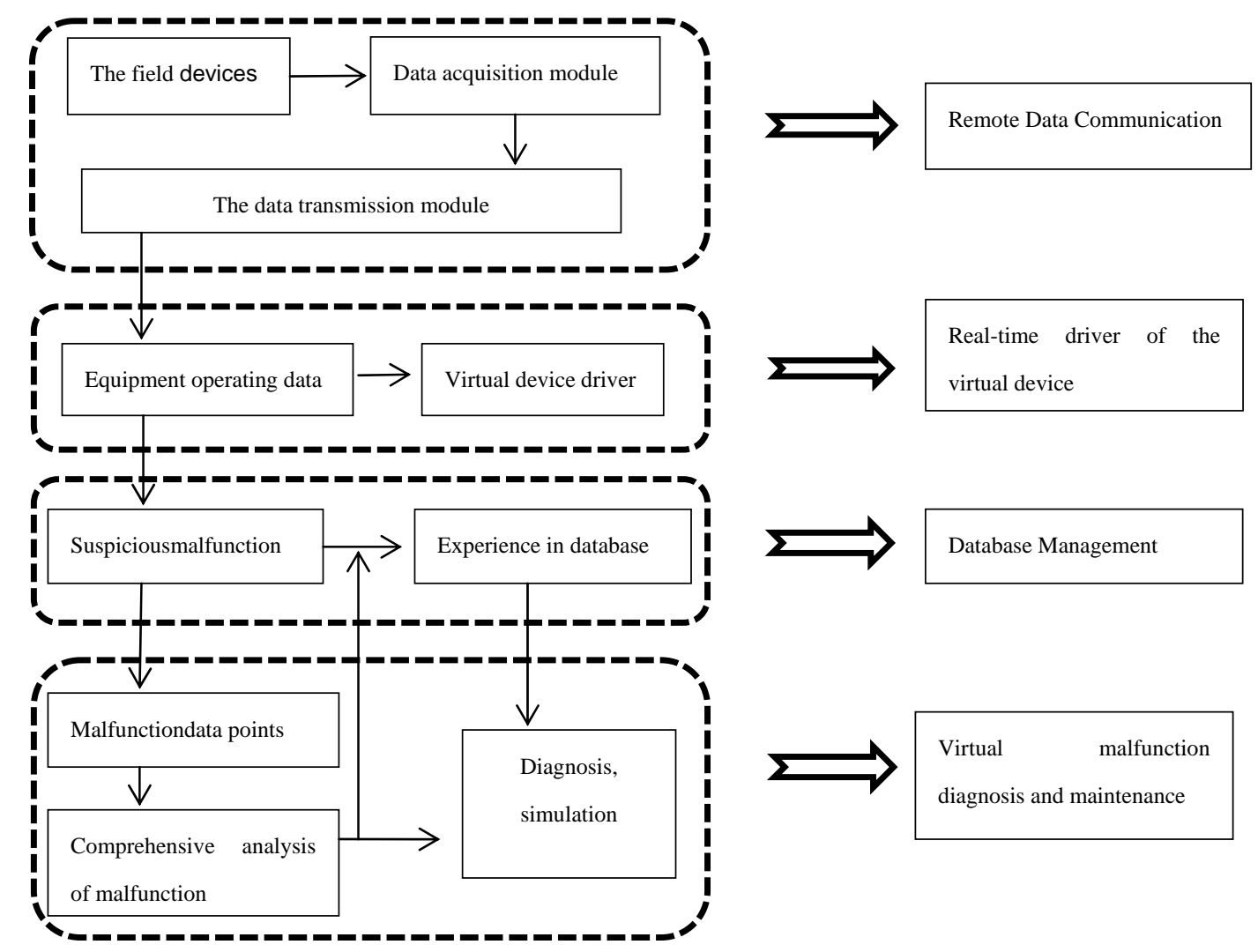

Figure 1. The function of system

\section{(1) Remote Data Communication}

Remote data communication functions to build a data communication link between the monitoring center with the terminal equipment, the operational status of data collection terminal equipment, PLC internal memory cell data. The same time, malfunction diagnosis, equipment maintenance needs of remote data communications link to reset the PLC internal parameters, or re-write the PLC program code. The middle of using the 3G network for data transmission, will greatly enhance the transmission rate, the data relay module and data module used for the acquisition of PLC data transmission to the 3G network terminal module, the module embedded TCP/IP protocol can be received PLC IP packaged into over 3G networks to remote manufacturing enterprise monitoring center data receiver module.

\section{(2) Virtual Device Driver in Real Time}

During the critical operating parameters, remote malfunction diagnosis, monitoring real-time access to remote data communication link terminal equipment to drive the virtual model of the monitoring center, so that the 
virtual device in the computer in real time to reflect the current operational status of the terminal equipment. When failures occur, according to the diagnostic results and contact database of experience in malfunction diagnosis system can quickly retrieve node information to the malfunction location, and drive the virtual device corresponding to the location of color, flashing, in order to achieve the purpose of locking the malfunction location.

\section{(3) Database Management}

Database management functions for the entire system, which includes the management of user information, device information management, malfunction management and failure to maintain control table information to the management of the report. Critical information which is stored in the database device malfunction diagnosis, such as the remote user rights, remote device communication address information, address information of the PLC storage unit, storage unit normal data value, the abnormal data and abnormal data corresponding to the cause of the malfunction, malfunction location, maintenance method, the database is one of the central part of the whole system, plays a very important role.

\section{(4) Virtualmal Function Diagnosis and Maintenance}

The process of malfunction diagnosis based on the acquisition of terminal equipment PLC data retrieval failure diagnosis experience database to get the suspected point of failure in the equipment, and the virtual device to output the location of the suspected point of failure. Which the failure to control the degree of perfection and accuracy of the table directly affect the system malfunction diagnosis capability. At the same time, a number of suspicious points of failure, malfunction diagnosis require a combination of reflected in the virtual device information, and ultimately determine the reasons for equipment failure and maintenance and, ultimately, the report output in the form of malfunction diagnosis to diagnosis.

Failure to maintain the process to resolve the failure of the remote device is based on the results of malfunction diagnosis. Maintenance of equipment failure can be divided into two cases: one is through the remote network can be solved; another cannot be solved through the remote network. In the former case, by the remote network to reset the parameter values of the terminal equipment PLC, or reload the PLC program. In the latter case, the failure approach, either by telephone guidance to solve or need to send technical support staff to the device to use the scene to solve.

\section{Key Technology of Virtual Diagnostic System}

\subsection{Experience in Database Design and Collaborative Sharing}

An on-site equipment maintenance program should be credited to the database, this is not only a valuable experience, which clearly favor the other technical staff as reference, but also the equivalent ofindividual cases have been established for each machinefile, if the frequency of maintenance of the equipment is greater than a reference value, the system will be warning enough time for the device processing to the enterprise, companies must consider the equipment to do the overhaul or scrap processing. Therefore, the design of the data and sharing of experience is essential.

Experience database design, including a few tables: Purchaser Info (equipment owner's information), the table is used to record the device has some information; Maintain Record (equipment maintenance records), the table is used to record equipment maintenance records; Remote Equipment (device specific information), the table used to store equipment for specific information; User Info (user information), which records the operating personnel of the different permissions, different permissions operator can look at the different database information; Alarm Log, (alarm history), recording equipment, maintenance frequency alarm information.

Different regions of the experts in the diagnosis and treatment of different devices, according to their competence to remotely log in to the system, so that by monitoring server on a remote device diagnostics, and the ability to share experience in the database solution.

\subsection{Real-time Driver of the Virtual Device Implementation}

This related to the virtual device driver in real time is divided into two cases: the first case is receiving monitoring center to the remote device's data, then parse through the data, access to reflect the relevant parameters of the device is running, such as the motor speed to drive the movement of the virtual device. Another situation is when the equipment malfunctioned remote malfunction diagnosis process to quickly lock the position of failure and be able to drive real-time display of virtual device malfunction location, the malfunction location. 


\section{A Virtual Device of the Motion Control}

\section{(1) The Operation of the Remote Device Data}

PLC for the control of the core equipment, the remote device access to data from the PLC internal, this result is directly related to this means of communication, the contents of the data communications will be discussed in detail in Chapter repeat them here. PLC for the control of the core machinery and equipment, to reflect the parameters of its operation parameters for the critical control site, such as the motor speed, sensor status and parameters are able to directly or indirectly reflect the actual operation of the device. To communicate with the remote PLC equipment directly from the PLC internal access to the information of these parameters may get information that can be used by the action unit to drive a virtual device by the parsing of the data.

\section{(2) Mapping of Virtual Devices Running Real-time Data-Driven}

A PLC controlled machine tools, for example, can reflect the operation of the machine parameters are: the speed of the machine tools, machine tool feed rate in X, Y. Direct access to the data parameters from the PLC machine motor speed, X-, Y-direction servo motor speed. Inside there is no such mapping:

a. Machine speed $=$ machine speed of the motor * transmission ratio

b. $\mathrm{X}$ direction feed rate $=\mathrm{X}$-direction motor speed $*$ gear ratio*coefficient

c. The Y-direction feed rate $=$ Y-direction motor speed*gear ratio*coefficient

By the above three relations, as long as access to the internal parameters of the PLC will be able to reflect the current operation of the device.

\section{(3) Virtual Device Driver}

With real-time access from the remote device parameter information, have a real-time mapping of data-driven virtual device running, you can determine the current operating conditions of the equipment. On virtual environment ofVRML, the idea of the event routing, parameter information is passed to the virtual device of the motor units; control unit node attributes change, in order to achieve the movement of the virtual device.

\subsection{Diagnosis Output}

Virtual remote malfunction diagnosis result output during the malfunction, there are two main ways:

(1) Virtual Device on the Malfunction Location

Malfunction location on the virtual device is output as a result in the computer processing stage processing is complete. Have dealt with the virtual device in real time to drive the implementation process, the virtual device in real time to drive the results, there are two, one is driven equipment operating data collected in real time the operation of the virtual device operating conditions of the virtual device to reflect the health of field devices; Another draw suspicious point of failure during the database search, display a suspicious point of failure in the virtual device location. The suspicious point of failure location in the virtual device, you can make the malfunction diagnosis through a visual interface to quickly understand the position relationship between the number of suspicious point of failure, drive the relationship and potential of the logical control relationship, malfunction diagnosis by virtue of these information analysis, evaluation, in order to arrive at a final diagnosis.

(2) The Output of Malfunction Diagnosis Report

Malfunction diagnosis to come to the final diagnosis, the report output malfunction diagnosis. Malfunction report is the completed by the engineers of the equipment malfunction diagnosis. A report should contain the exception data collected, the suspected point of failure information, diagnosis information and other important information. In addition, the malfunction diagnosis report or the basis for further failure to maintain, so it should also contain a detailed breakdown maintenance instructions, such as equipment maintenance, and need further testing parts need to re-set parameters, the malfunction diagnosis problem approach, the need to send technical support staff, and other information.

\section{Conclusions}

The paper from the actual application examples proves the feasibility of the tool presses PLC terminal equipment data acquisition. Based on the $3 \mathrm{G}$ network of wireless communications, remote diagnostics is no longer subject to geographical constraints. It discusses the modeling of the virtual device prototype and drive virtual prototype by controlling real time action units. Designing system database, virtual remote malfunction diagnosis unites repair mode and active diagnostic patterns.Real-time drive technology of virtual prototype which isused in the process of remote malfunction diagnosis, help troubleshooters analyzes the failure. A comprehensive analysis of 
the above information is in order to quickly get the reason of the failure of the equipment. It is a good application tosolve the printing presses of remote malfunction diagnosis.

\section{References}

Chen, L. D., \& Feng, J. H. (2010). Design of remote environmental monitoring system based on PLC and GPRS. Automation and instrumentation, 26, 28.

Kang, J., \& Luo, J. M. (2006). Based VPN device for remote maintenance and diagnosis method. Computer Information, 23, 24.

Li, J. B. (2010). Virtual manufacturing system action reproduction technology. University of Shanghai for Science and Technology.

Liu,Z. X.,Yu, Z., \& Chen, Y. P. (2007). GPRS DTU communication of data center design. Industrial control computer, 20(12), 23-26.

Sun, Y. N., \& Fu, J. Y. (2010). Temperature controlling design based on VB and PLC. Information Theory and Information Security,134, 137. 